\title{
Finite-size effects in the dynamics and thermodynamics of two-dimensional Coulomb clusters
}

\author{
E. Yurtsever \\ Koç University, Rumelifeneriyolu, Sariyer, Istanbul 34450, Turkey \\ F. Calvo \\ Laboratoire de Physique Quantique, IRSAMC, Université Paul Sabatier, 118 Route de Narbonne, F31062 Toulouse Cedex, France \\ D. J. Wales \\ University Chemical Laboratories, Cambridge CB2 1EW, United Kingdom
}

(Received 31 January 2005; revised manuscript received 26 April 2005; published 11 August 2005)

\begin{abstract}
The dynamics and thermodynamics of melting in two-dimensional Coulomb clusters is revisited using molecular dynamics and Monte Carlo simulations. Several parameters are considered, including the Lindemann index, the largest Lyapunov exponent, and the diffusion constant. In addition to the orientational and radial melting processes, isomerizations and complex size effects are seen to occur in a very similar way to atomic and molecular clusters. The results are discussed in terms of the energy landscape represented through disconnectivity graphs, with proper attention paid to the broken ergodicity problems in simulations. Clusters bound by $1 / r^{3}$ and $e^{-\kappa r} / r$ forces, and heterogeneous clusters made of singly and doubly charged species, are also studied, as well as the evolution toward larger systems.
\end{abstract}

DOI: 10.1103/PhysRevE.72.026110

PACS number(s): 64.60.Cn, 68.65.-k, 36.40.Sx

\section{INTRODUCTION}

The finite size analogs of two-dimensional (2D) Wigner crystals have received considerable attention from theoreticians [1-16], and more recently also from experimental groups [17-21]. Particles in mutual repulsion can be confined using a variety of methods, most importantly electrostatic traps of the Paul or Penning types, and simple hard walls, usually of circular shape. The repulsion between particles depends upon the details of the experiment. Arrays of electrons on the surface of liquid helium [22] or in quantum dots [23] can be described by conventional Coulomb forces. However, logarithmic forces are more relevant for vortex clusters in superfluids [24] or Bose-Einstein condensates [25]. Mesoscopic colloidal particles undergo dipolar $1 / r^{3}$ interactions within an external magnetic field [26], or a Yukawa (screened Coulomb) potential when they are charged and placed in a viscous medium.

Finite 2D assemblies have also been synthesised on a more macroscopic scale, using metal particles close to $1 \mathrm{~mm}$ in size [19]. In this study, the authors were able to visualize and locate not only stable configurations, but they could also estimate the shape of the transition states, which are here defined as stationary points with Hessian index one [27].

Another example of experimentally accessible Wigner crystals occurs for dusty plasmas. These highly charged particles of micrometer size and charges up to $Z=10^{4}$ can be stabilized as gaseous plasmas. The gravitational forces and the electric field balance each other, so that the dust particles form highly regular two-dimensional structures. By changing the electric field, or conversely the pressure, of the plasma, transitions from solid to liquid-like phases are observed [17]. A two-stage melting was proposed, which does not follow the predictions of two-dimensional melting theories, but rather goes through an intermediate phase. This phase has been described as an oscillating crystal [21]. Finally, by mea- suring the spectra of dust particles, it has been suggested that the dynamics may be a size-dependent phenomenon, that is, the effects of various types of motion such as intershell rotation and breathing may play different roles [20].

A major part of our theoretical understanding of the above systems comes from numerical studies. The static properties of the assemblies were obtained using optimization methods ranging from simulated annealing to genetic algorithms, and a Mendeleev-type table was proposed by several authors $[1,5]$ to reflect the special stability of certain sizes. Comparisons with the theoretical predictions of the "Thomson atom" [28] were also carried out [6]. Shell effects were more generally investigated semianalytically by Koulakov and Shklovskii [11]. Metastable configurations (excited states) and the potential energy barriers between them have been studied in Refs. $[4,12,15,29]$. Topological defects were also identified for stable structures [13].

Melting in 2D Coulomb clusters has been a subject of debate. The results of the classical and quantum Monte Carlo simulations of Lozovik and Mandelshtam [2] were interpreted as a two-step melting process, where orientational melting occurs prior to radial melting. In this process, shells remain quasicrystalline, but still undergo some concerted rotational motion. Radial melting conversely involves exchanges between particles of adjacent shells. This two-step process is generally accepted for small assemblies, but it is not clear whether, in large clusters, the external shell may also exhibit orientational melting [12] or not [9].

Several factors are likely to influence the stability of 2D Coulomb clusters. First, rotational barriers are strongly dependent on the structure, which in turn depends on the size. More stable structures are obtained when the core is based on a triangular lattice, thus resembling most observed Wigner crystals. More importantly, the very different nature of orientational and radial melting has led to some confusion about how suitable observables should be defined to probe these 
processes separately $[14,30,31]$. In their study of binary 2D clusters, Drocco et al. [16] chose an order parameter based on the polar angles of the particles in order to quantify the extent of orientational motion in Langevin molecular dynamics simulations. However, this measure becomes impractical as soon as a central particle is present, as it then becomes very noisy.

Melting in atomic and molecular clusters also displays a very rich phenomenology [32]. In particular, surface melting [33], plastic transitions [34], or anomalously high melting temperatures [35] have been identified from experiments or simulations. For three-dimensional trapped-ion clusters, the structures are sometimes related to those found in atomic clusters [29]. The study of atomic clusters also shares with Coulomb assemblies the lack of a proper universal order parameter to characterize the melting point. For instance, the Lindemann criterion for melting does not always provide useful information in the case of solid-solid transitions and premelting effects [36].

The largest Lyapunov exponent $\lambda$, which quantifies chaos and the sensitivity to initial conditions, is a useful tool for probing the complex dynamics of atomic and molecular clusters [37-41], especially at melting. Because Lyapunov exponents are related to the second derivatives of the potential energy surface (PES) [42], we expect them to be sensitive to orientational melting.

In this paper, we revisit the dynamics and thermodynamics of 2D Coulomb clusters by focusing on dynamical measures such as $\lambda$ or the diffusion coefficient, $D$, and by performing extensive Monte Carlo simulations employing the parallel tempering strategy [43]. We also consider the interplay between the dynamics and the energy landscape of these clusters by constructing disconnectivity graphs $[44,45]$. Finally, we investigate how the thermodynamic behavior evolves with size.

This paper is organized as follows: After briefly presenting our methods, we choose some specific examples to illustrate the difficulties associated with an unambiguous definition of the melting point in these clusters, and we then discuss the large size variations. We summarize our conclusions in Sec. IV.

\section{METHODS}

Two-dimensional Wigner clusters are characterized by the following classical potential energy:

$$
E(\mathbf{R})=\sum_{i<j} \frac{q_{i} q_{j}}{r_{i j}^{p}} \exp \left(-\kappa r_{i j}\right)+\sum_{i} A r_{i}^{2} .
$$

In this equation, $\left\{q_{i}\right\}=1$ or 2 are the charges carried by the particles, $r_{i j}$ is the distance between particles $i$ and $j$, and $r_{i}$ is the distance from particle $i$ to the cluster center-of-mass. The parameter $p$ will usually be 1 (Coulomb case), but we also specifically studied the case $p=3$ (dipolar interaction) for which some results will be selected below. The range of the electrostatic potential, $\kappa$, was set to 0 (pure Coulomb) or to a finite value (shielded Coulomb). Finally, and for comparison with the work reported in Ref. [16] on binary clusters, we have taken $A=10$. It should be noted that, in the absence of the exponential term, the physics of the system is independent of $A$ : under the transformation $(r, E)$ $\rightarrow\left[r A^{1 /(2+p)}, E / A^{p /(2+p)}\right]$, the energy can be cast in a form that does not depend on $A$. In the following presentation, unit masses and charges are used and all properties are explicitly given in dimensionless reduced units.

The global minima of the clusters were investigated using the Monte Carlo plus minimization algorithm, also known as basin-hopping $[46,47]$. The 2D clusters can generally be described as multishell systems, and we will use the $\left(n_{1}, n_{2}, \ldots\right)$ notation for a homogeneous cluster having $n_{i}$ particles in its $i^{\text {th }}$ shell. Binary clusters will be referred to as $\left\{N_{s}, N_{d}\right\}$, where $N_{s}$ and $N_{d}$ are the respective numbers of singly and doubly charged particles. Due to the relatively long range of the potential [27], global optimization is relatively straightforward for Coulomb systems.

Beyond the stable isomers, we also investigated higherindex stationary points on the energy landscape. Locating the saddle points with Hessian index one enabled us to construct disconnectivity graphs [27], in order to relate the observed dynamical and thermodynamical behavior to the potential energy surface for these clusters. We refer the reader to Ref. [27] for further details of how stationary points are located and disconnectivity graphs are generated.

The dynamics has also been studied by solving Hamilton's equations of motion at constant energy. The coupled equations were integrated by the four-step Runge-Kutta method, which keeps the energy constant to at least seven digits. For the first trajectory, the initial positions of the particles were obtained from the global minimum structure and a random set of momenta were chosen. The momenta were then scaled to set the linear and angular momentum to zero and the vibrational temperature to around $10^{-7}$. The time step was taken to be 0.0025 . The first $10^{5}$ steps were used to equilibrate the system and discarded, then $10^{6}$ steps were used for each simulation. Further trajectories start with the configuration obtained from the previous run, with velocities scaled approximately to give a new temperature increased by a factor 1.25. Again, the equilibration steps were discarded.

For each trajectory the average kinetic temperature, rootmean-square (RMS) bond-length fluctuation index $\delta$, the maximal Lyapunov exponent (MLE) $\lambda$, and diffusion coefficient $D$ were calculated. $\delta$ is commonly used to detect melting in bulk or finite (3D) atomic systems, as it is expected to jump from a low value to around 0.1-0.15. Both the diffusion coefficient and the Lyapunov exponent were calculated from shorter parts of the entire trajectory. $D$ was obtained from the slope of the mean square atomic displacement. This measure describes the breathing and inter-shell exchanges (isomerizations), but does not identify even qualitative changes in the rotational structure. For homogeneous systems it is very difficult to define measures of the angular motion. Since the total angular momentum is zero, one can only measure angular velocity and momenta for separate shells; however, the shell definitions lose their significance once isomerizations start taking place. In addition, the angular properties become ill-defined for clusters having a central particle.

The calculation of the MLE was motivated by several studies indicating possible relations between phase changes 
and this dynamical indicator. The MLE measures the sensitive dependence on initial conditions and is defined from the exponential separation of two trajectories that start infinitesimally close together in the phase space. A tangent space approach was used in the numerical calculations [48]. However, we should mention that semianalytical theories have also been proposed to quantify the MLE from the statistical properties of the potential energy surface [49].

The equilibrium thermodynamics of the 2D clusters was also studied by means of parallel tempering Monte Carlo (MC) simulations. Parallel tempering is a particularly efficient method to accelerate convergence in systems whose natural dynamics is affected by broken ergodicity. Most often such problems are related to multiple funnels [27] or "glassy" energy landscapes. The MC simulations were performed in the canonical ensemble, with temperatures in the range $10^{-5}<T<10$. About 80 runs were carried out in this range, with the temperatures nearly equally spaced on a logarithmic scale. $10^{5} \mathrm{MC}$ cycles were used for equilibration, followed by $10^{6} \mathrm{MC}$ cycles for the actual calculations. The $\mathrm{MC}$ calculations were principally used to compute the thermal properties, such as the heat capacity, $C_{v}$.

In both MC and MD simulations the relation between the energy and the temperature is quasilinear.

\section{RESULTS}

The snapshots obtained from experiments on colloidal particles [19] or dust plasmas [20,21], as well as the various molecular dynamics (MD) simulations, exhibit three types of characteristic motion. At low energy or low temperature, or for dust plasmas under high pressures, only small amplitude motion is observed, in which the particles oscillate around their mean positions. As the energy increases, these oscillations gain enough momentum to achieve complete rotations in their respective shells. The directions of these rotations are random, and they may change over time. However, in order to preserve the total angular momentum, different shells must rotate in opposite directions. Eventually, actual isomerizations between shells may take place, as previously examined in 3D systems [29]. In one case, it has also been reported that isomerizations may occur before the full rotational motion is achieved [20]. We expect this observation to be due to rather infrequent sampling of the experimental trajectories [20]. It is clear that the angular and the radial motion of particles contribute differently to all the measures aforementioned.

In order to study the mechanism of phase transitions in 2D clusters, we have carried out simulations of homogeneous clusters systems in the size range $3 \leqslant n \leqslant 40$. We selected several sizes displaying different but typical characteristics. In Fig. $1, \lambda, D, \delta$, and $C_{v}$ are plotted with respect to the average temperature for sizes $17,21,22$, and 30 . The global minima for these clusters are found to be $(1,6,10)$, $(1,7,13),(2,8,12)$ and $(5,10,15)$, respectively, in agreement with Ref. [3]. The MLE starts becoming nonzero at very low temperatures, around $T=10^{-4}$. In this regime, the dynamics are more irregular for $n=17$ and $n=21$. Similarly, the Lindemann index and the diffusion coefficients are also higher for $n=17$ and $n=21$ in the low $T$ regime. Both these clusters exhibit an intermediate phase over a broad temperature range (note the logarithmic temperature scale). On the other hand, the 30-particle cluster exhibits a single phase transition.

While the three dynamical indicators agree with one another, the heat capacities provide little information in comparison. The general behavior observed for all sizes is that of harmonic oscillators at very low temperatures $\left[C_{v} \rightarrow(2 n-3) k_{B}\right]$ or of free, independent particles at high temperatures $\left[C_{v} \rightarrow(2 n-3) k_{B}\right.$ again due to confinement]. In the intermediate temperature range, a strong decrease is seen near $T \sim 2$ for all sizes, following an order-disorder peak below $T=0.1$. The curves for a specific cluster are built on the basis of this generic behavior, which reminds us of the caloric curves computed for Lennard-Jones polymers [50]. For some sizes, such as $n=21$ or $n=30$, extra bumps or shoulders occur as a consequence of isomerizations. In general, the strong increase of $\lambda, D$, or $\delta$ at $T \simeq 0.03$ correlates with a peak in the specific heat. However, the phenomena responsible for the peculiar dynamical features for the 17and 21-particle clusters do not have any thermal signature. The premelting heat capacity peak seen for $n=21$ at $T$ $=0.01$ does not have any counterpart in the dynamical indices either.

These data can be interpreted using the disconnectivity graphs shown in Fig. 2. In this figure, saddles connecting two permutations of the same isomers have been included to account for the rotational barriers. Such saddles are manifested by the presence of "copies" of the corresponding minima on the graph.

The energy barriers, which link the global minimum to itself through an internal rotation, are much lower for $n$ $=17\left(\Delta E=4 \times 10^{-6}\right)$ and $n=21\left(\Delta E=10^{-9}\right.$, this is nearly a case of free rotation) than for larger clusters, where they are closer to $\Delta E=10^{-3}$. Such degenerate rearrangements [27] do not play a major role in thermal equilibrium, but can have important dynamical consequences. This is precisely what we observe in Fig. 1. The barriers for rearrangements are much higher in energy than those involved in the rotational motion, but in some cases other stable isomers lie relatively close to the global minimum. For $n=21$ and $n=30$, such isomerizations are indeed seen and correlate with bumps in the canonical heat capacity.

From the previous results it is clear that one cannot draw general conclusions by looking at dynamical indicators alone, or at observables aimed at characterizing thermal equilibrium. We have also looked at two specific cluster sizes, for which the results of the pure Coulomb case have been compared to other interaction forms. The 31-particle cluster was studied with both $1 / r$ and $1 / r^{3}$ interactions, the latter expression being more relevant to colloidal particles, such as the ones involved in the experimental setup of Ref. [19]. The 42-particle cluster has recently been the focus of an experimental report on dust plasmas [21]. In this situation, a screened Coulomb or Yukawa form $e^{-\kappa r} / r$ is more appropriate than the bare Coulomb law. Here we chose $\kappa=1$, but the results presented hereafter in Fig. 3 are not significantly different for other values in the range $0.1 \leqslant \kappa \leqslant 10$.

We first discuss the 31-particle clusters, which display different behavior depending on the form of the interaction. 
(a)

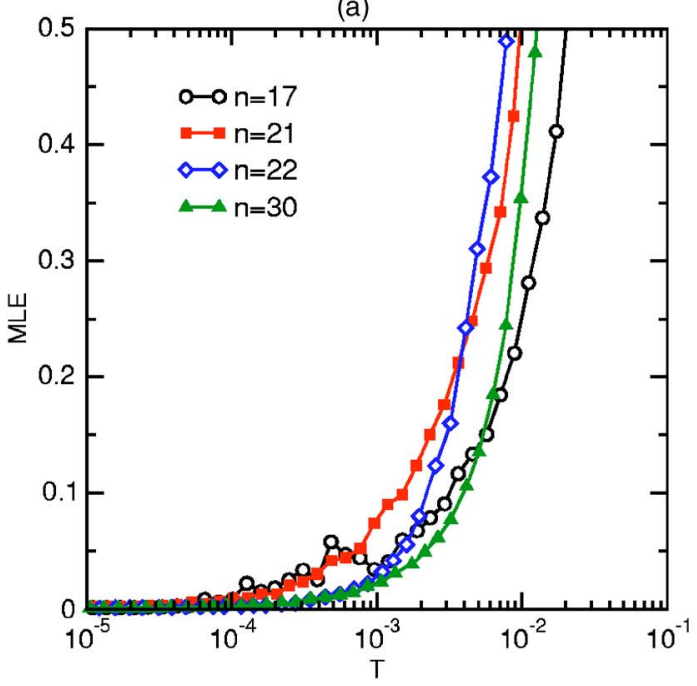

(c)

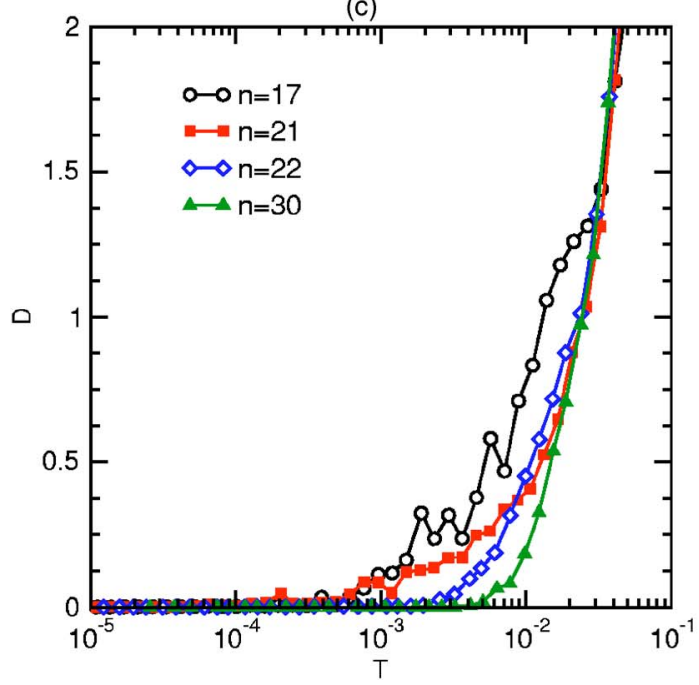

(b)

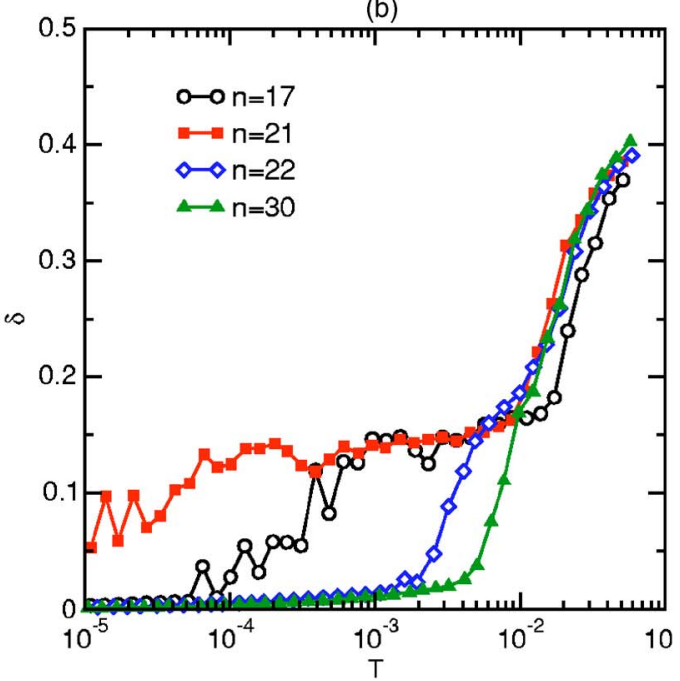

(d)

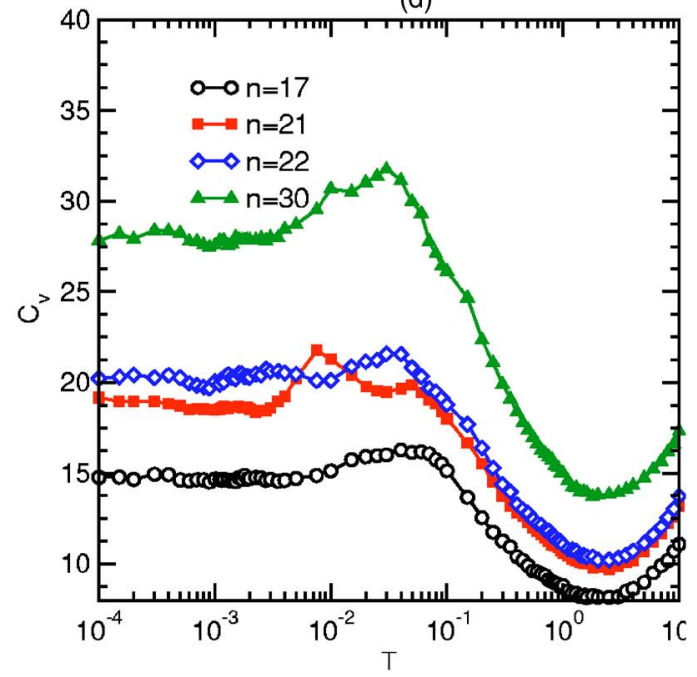

FIG. 1. (Color online) Dynamic and thermodynamic properties of some homogeneous 2D Coulomb clusters with $n=17,21,22$, and 30 as a function of temperature. (a) Maximal Lyapunov exponent (MLE) $\lambda$; (b) RMS bond-length fluctuation $\delta$; (c) diffusion coefficient $D$; (d) canonical heat capacity $C_{v}$. The data in (a) to (c) are from microcanonical MD simulations; the data in (d) are from canonical Monte Carlo simulations. $C_{v}$ is in units of the Boltzmann constant.

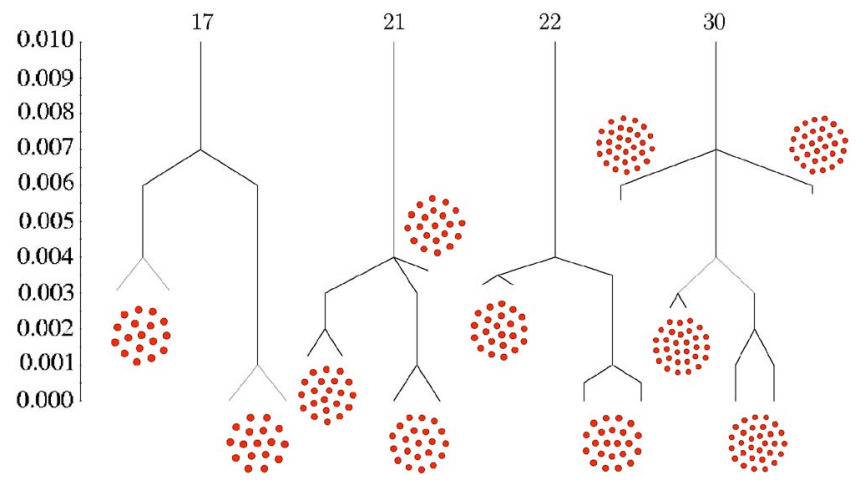

FIG. 2. (Color online) Disconnectivity graphs for the homogeneous clusters of Fig. 1. The global minimum energy is shifted to zero for each size.
While the Coulomb potential leads to a $(5,11,15)$ three-shell global minimum, the shorter range dipolar interaction favors a $(1,5,10,15)$ ground state. Because this structure has a central particle, the vibrational motion is more constrained, and the harmonic entropy of $(5,11,15)$ is actually larger. This competition between energy and entropy leads to a structural transition, which is seen in the heat capacity of Fig. 3(a) as a significant peak near $T=0.03$, while the melting peak is located at $T \sim 0.5$. The Coulomb cluster behaves very differently, with a single $C_{v}$ peak at $T=0.03$. Scrutiny of the Lindemann index indicates that this cluster undergoes free internal rotation at very low temperatures, $T<10^{-4}$, before fully melting at $T=0.03$. In the case of the dipolar interaction, only a single, but very sharp, increase is seen, at temperatures even higher than the melting peak, $T \sim 0.1$. This surprising result has been checked and confirmed by extensive sampling. After regular quenching of the MD trajectories in this temperature range, we found that the sharp in- 
(a)

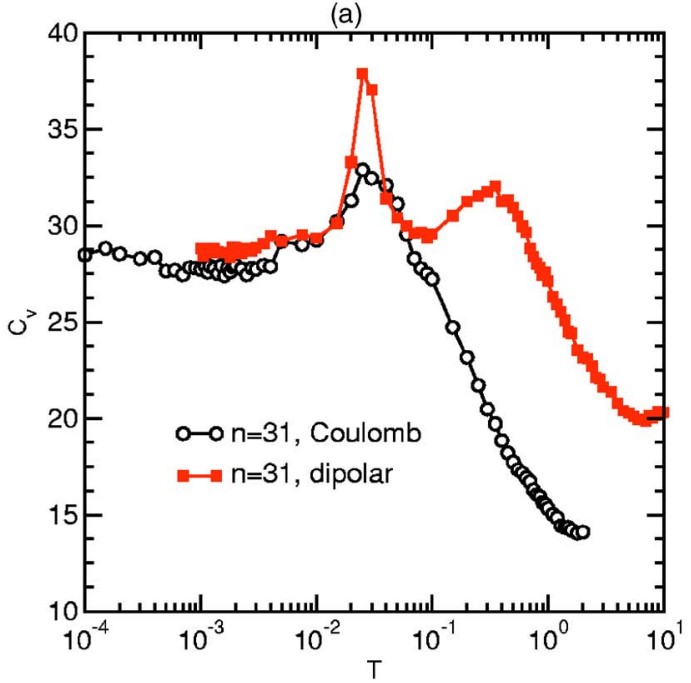

(c)

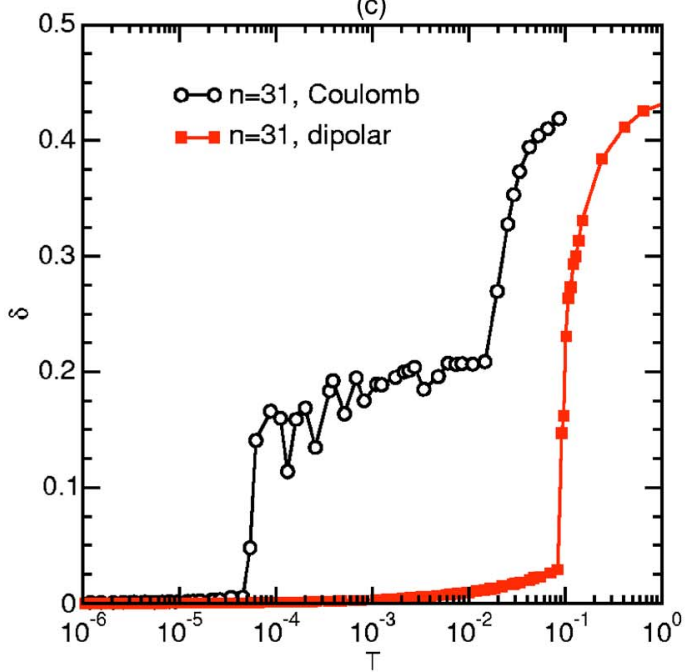

(b)

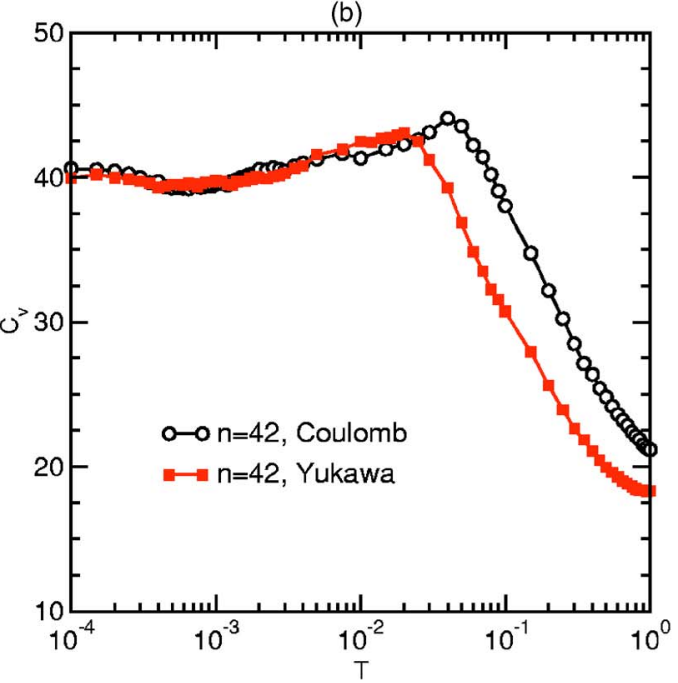

(d)

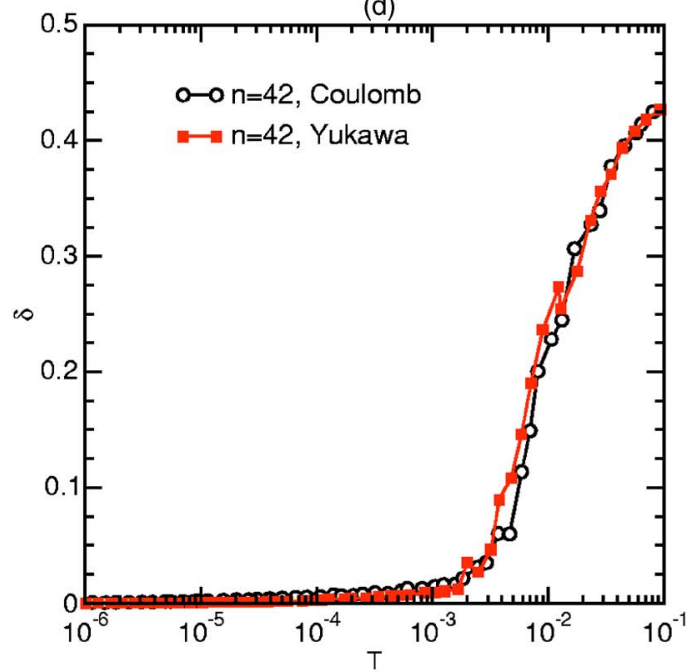

FIG. 3. (Color online) (a) and (b) Heat capacities of 31- and 42-particle clusters, respectively (in units of the Boltzmann constant). (c) and (d) RMS bond length fluctuation indices. For $n=31$, the results of the Coulomb $(1 / r)$ and dipolar $\left(1 / r^{3}\right)$ interactions are shown. For $n$ $=42$, the results obtained with the Yukawa potential $\left(e^{-r} / r\right)$ are compared to those with the bare Coulomb form.

crease of $\delta$ was correlated with a sudden isomerization to the $(5,11,15)$ second-lowest minimum, which is also involved in the preliminary structural transition. The lowest energy transition state from the global minimum was found to be significantly higher than the energy of the second isomer. In such a situation, MD simulations suffer from broken ergodicity, since they cannot sample the available phase space until sufficient energy is added. In cluster physics, a wellstudied example of broken ergodicity is provided by the 38atom Lennard-Jones cluster [51,52]. The present sharp jump of the Lindemann index should not be interpreted as the onset of melting, but rather as a signature of isomerization.

42-particle clusters were investigated with the aim of finding some thermodynamic confirmation that melting proceeds in multiple steps. Unfortunately, neither the specific heats of Fig. 3(b) nor the Lindemann indices of Fig. 3(d) show any multiple-stage behavior that could be related to the experimental observation [21]. We thus conclude that the dust plasma system studied by Melzer and co-workers can- not be simply modeled as if it were made of a single layer.

Comparing the general curves for the Coulomb, dipolar, and Yukawa potentials also provides useful information about the factors influencing the melting point. Yukawa systems exhibit a significant shift of their melting temperature toward lower $T$. Since the Yukawa potential is an attenuated form of the pure Coulomb form, this is the expected trend. At large distances, the dipolar interaction is also much smaller than the Coulomb interaction. However, the melting temperature of Coulomb clusters is about one order of magnitude smaller than for the dipolar cluster. Hence the longrange part of the potential plays opposite roles in the dipolar and Yukawa forms. This result suggests that the location of the melting peak is mainly driven by the short-range repulsive part of the potential.

We now turn to small, binary Coulomb clusters, such as those recently investigated by Drocco and co-workers [16]. $N_{s}$ particles with charge 1 and $N_{d}$ particles with charge 2 are stabilized in a parabolic trap with $A=10$. The structures again 
(a)

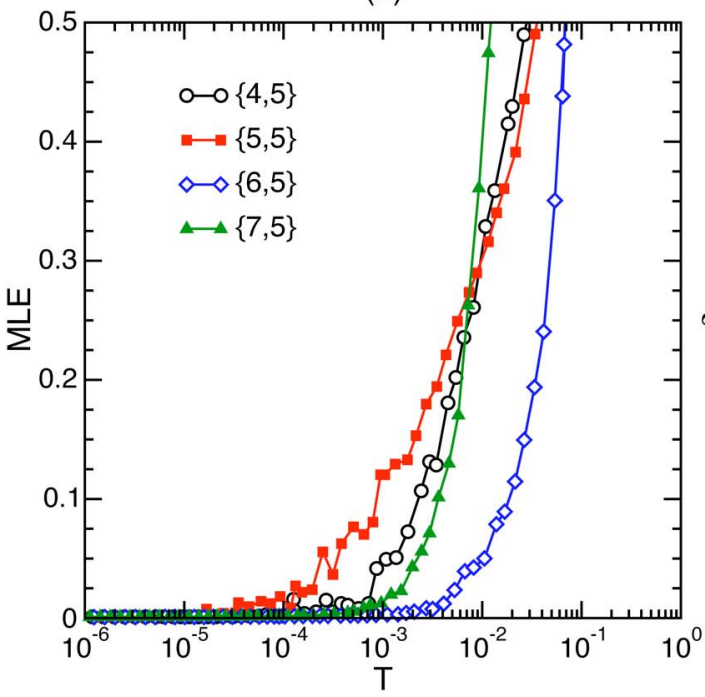

(c)

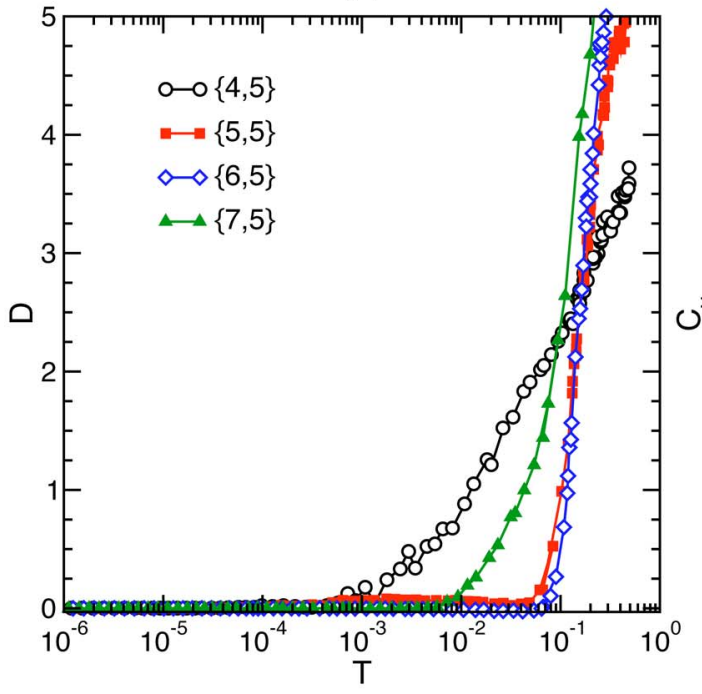

(b)

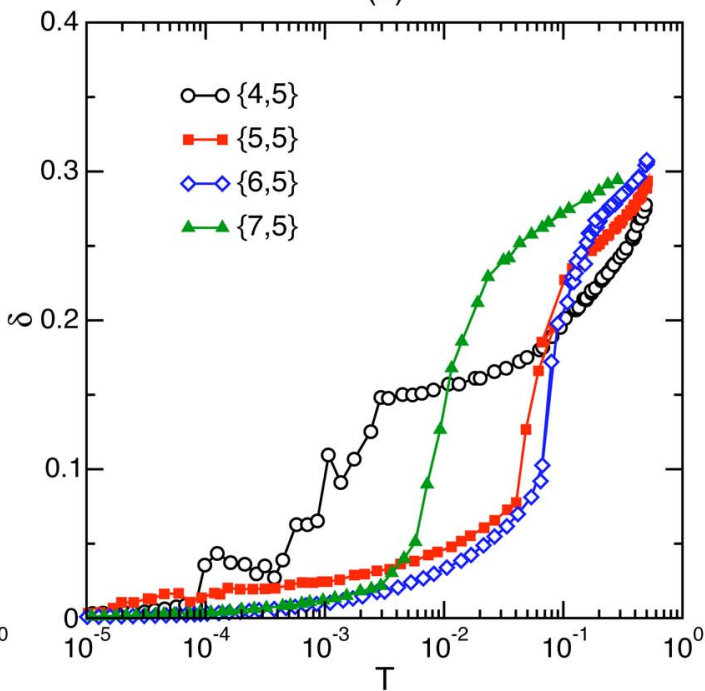

(d)

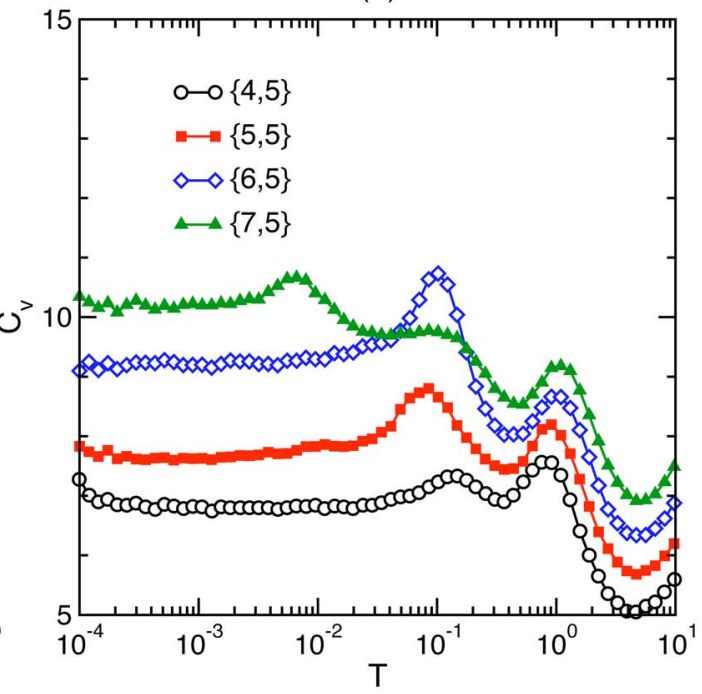

FIG. 4. (Color online) Dynamic and thermodynamic properties of some binary $\{n, 5\} 2 \mathrm{D}$ Coulomb clusters with $n=4-7$ versus temperature. (a) Maximum Lyapunov exponent (MLE) $\lambda$; (b) RMS bond length fluctuation $\delta$; (c) diffusion coefficient $D$; (d) canonical heat capacity $C_{v}$. The data in (a) to (c) are from microcanonical MD simulations; the data in (d) are from canonical Monte Carlo simulations. $C_{v}$ is in units of the Boltzmann constant.

consist of concentric shells where the singly charged particles form the inner shells in order to minimize repulsion. Drocco et al. used Langevin MD simulations for mixed clusters containing $N_{s}=3-34$ singly charged and $N_{d}=5,6$, and 7 doubly charged particles, and they also determined the melting points. These authors concluded that the melting behavior of these binary trapped particles strongly depends on whether the numbers of particles in the inner and outer shells are commensurate. They also found that the highest melting points were obtained for $N_{s}=N_{d}+1$, where the corresponding structures all have a single particle at the center.

We have performed MD simulations for these heterogeneous systems under similar conditions to those of the homogeneous clusters. In Fig. $4, \lambda, D, \delta$, and the specific heat $C_{v}$ are plotted for $\{4,5\},\{5,5\},\{6,5\}$, and $\{7,5\}$, selected as further typical examples, highlighting differences in the stability and dynamics. The onset of chaos occurs at tempera- tures close to those seen in the homogeneous systems. $\{6,5\}$ is the most stable cluster, in the sense that it has the highest melting point in Ref. [16], while $\{5,5\}$ is the most irregular. Both the diffusion and Lindemann indices show the same qualitative trends, namely that the melting points, as measured by the strong increase in $\delta$, follow the order $\{4,5\}$ $<\{7,5\}<\{5,5\}<\{6,5\}$.

As for homogeneous clusters, the variation of the heat capacities does not exactly match the dynamical indices. Here the generic shape again starts at a constant value at low temperature, which corresponds to harmonic behavior. The high-temperature gas phase is still characterized by the same constant value. In the intermediate temperature range, two main peaks are associated with the melting of successive subclusters made of singly charged, then doubly charged particles. Nearly one order of magnitude separates the two associated melting temperatures. This interpretation was fur- 


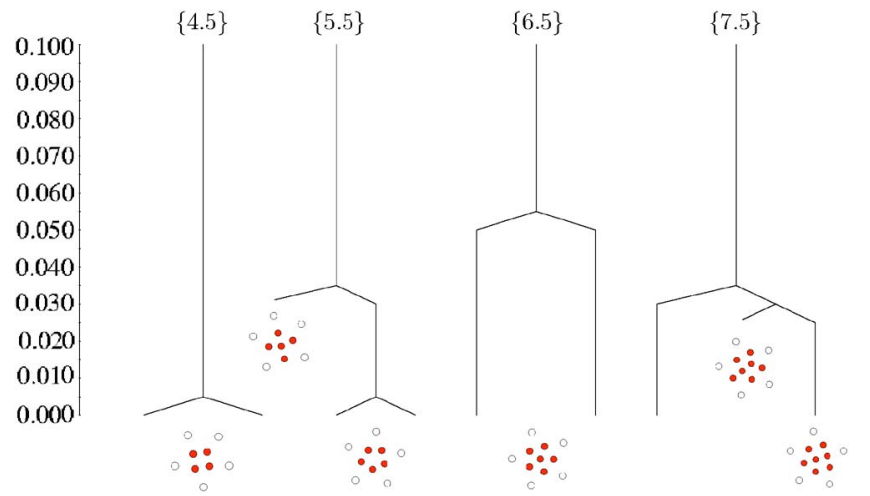

FIG. 5. (Color online) Disconnectivity graphs for the binary clusters of Fig. 4. The global minimum energy is shifted to zero for each size.

ther confirmed by calculating the heat capacity of the ternary $\{7,6,6\}$ cluster made of seven singly charged particles, six doubly charged particles, and six triply charged particles. For this cluster, three clear features are observed in $C_{v}$, and only above $T \sim 2$ do the triply charged particles mix with the rest of the cluster.

On top of this generic behavior, a specific isomerization shoulder is seen for the heat capacity of the $\{7,5\}$ cluster in Fig. 4(d), at the same temperature $T \simeq 0.01$ where the three dynamical parameters increase significantly. Again, the free rotation of some internal shells revealed by $\lambda, D$, and $\delta$ at low temperature has no thermal signature. The disconnectivity graphs for these clusters are shown in Fig. 5. As is particularly obvious from the graphs for $\{5,5\}$ and $\{6,5\}$, commensurate structure between the singly and doubly charged shells is not the only factor needed to explain the complicated behavior exhibited by these finite Wigner crystals. The extremely low barriers for the $\{4,5\}$ and $\{5,5\}$ clusters permit facile rotation, which is correlated with the rapid increase of all dynamical indicators at low temperatures. The $\{6,5\}$ cluster is especially resistant to isomerization because an extra, central particle hinders the radial motion of the particles in the other shells. Only for the $\{7,5\}$ cluster is the isomerization energy low enough to give rise to an extra peak in the heat capacity. This case, and the case of the single-isomer $\{6,5\}$ cluster, further show that the multiple-peak structure of the heat capacity is not a consequence of isomerizations. Therefore the general, two-peak shape of the heat capacity is not related to isomerization, but is intrinsic to the potential itself and the presence of two types of particles organized in shells.

The variation of the melting temperature with cluster size is shown in Fig. 6 for the three $\{n, 4\},\{n, 5\}$, and $\{n, 6\}$ binary series, with $2 \leqslant n \leqslant 20$. Here the melting temperature was defined according to the Lindemann criterion as the temperature where $\delta$ sharply increases above 0.1 . The variation of the computed melting point is qualitatively similar to that reported by Drocco and co-workers [16]. In particular, the high values for $\{4,4\},\{5,5\},\{6,5\}$, and $\{7,6\}$ are reproduced. However, our results quantitatively differ from those of Drocco et al. by a factor of at least 10 . Our data, obtained with exactly the same Hamiltonian as in Ref. [16], are in

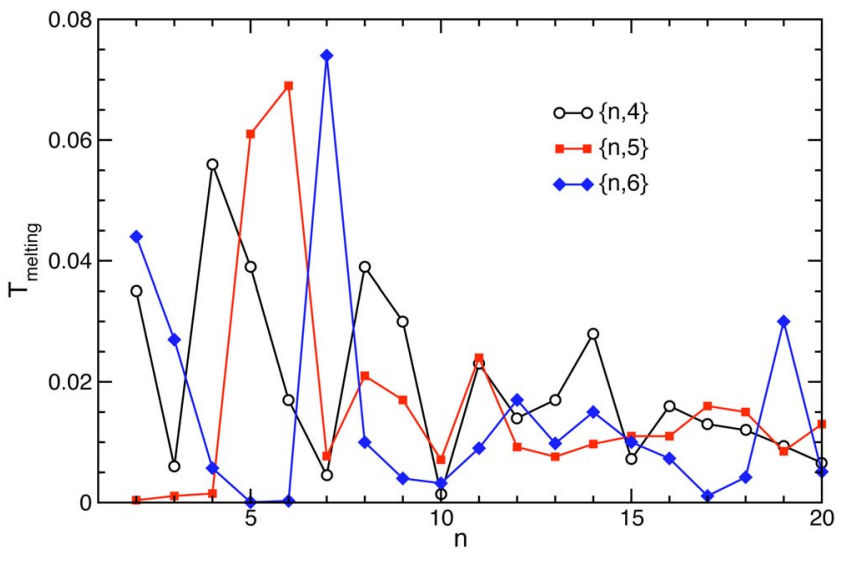

FIG. 6. (Color online) Size evolution of the melting point of the $\{n, 4\},\{n, 5\}$, and $\{n, 6\}$ series of binary Coulomb clusters as obtained from the Lindemann criterion.

agreement with the computed melting points for the homogeneous clusters, which are comparable with previous results for the same systems [3]. Even though the present temperatures result from time averages in our MD simulations, their range matches the canonical temperature of our MC runs. Hence we believe that the data of Fig. 6 are reliable, and that the differences from the results obtained by Drocco and coworkers are the consequence of an unreported alternative choice of $A$ in the Hamiltonian used in Ref. [16].

Lastly, we discuss the behavior of larger clusters and how melting evolves toward bulk. In Fig. 7(a) we show the heat capacities calculated from MC simulations for the cluster sizes $n=100,200$, and 500. The general trend is that the main peak near $T \simeq 0.04$ becomes narrower and sharper, suggesting that melting is a first-order process rounded by finite size effects [53]. However, the main heat capacity peak remains quite broad, so a more detailed study should probably be performed on even larger sizes, including a proper finite-size scaling analysis. In addition, extra features in the heat capacity are present at low temperatures, even for the largest 500particle cluster. Regular quenching confirms that these premelting effects are due to isomerizations involving different arrangements of the inner shells. At large sizes, even though the global shape of the cluster is roughly circular, the radial ordering into shells is replaced by a triangular close-packed lattice, possibly with several defects [13]. Rotation of the various shells then becomes hindered, and the dynamics is reduced to more usual consecutive isomerizations.

Figure 7(a) supports the idea that a single, first-order phase transition should occur in the bulk limit, if the limit could be defined. Since there is no unified theory of 2D melting [54], we should probably be cautious in extrapolating the heat capacities computed from Monte Carlo simulations. If we choose the peak of the heat capacity as the signature of melting, the melting point thus defined shows very pronounced variations with the cluster radius $n^{1 / 2}$, as illustrated in Fig. 7(b).

The data for all sizes in the range $5 \leqslant n \leqslant 50$ and for larger clusters $(n=60,70,80,90,100,120,140,160,180,200$, $250,300,350,400,450,500)$ are included in this graph. The strong size dependence for small systems is similar to that 

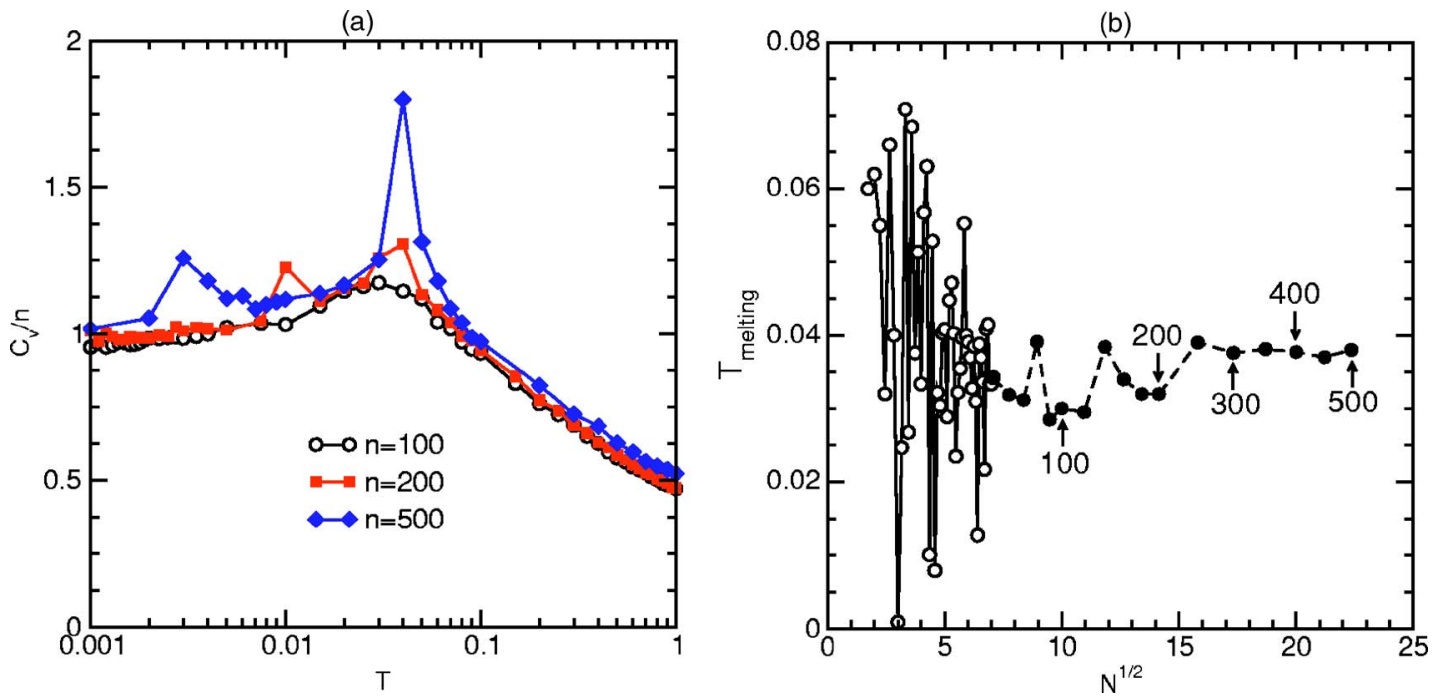

FIG. 7. (Color online) (a) Canonical heat capacities (in units of the Boltzmann constant) of some large homogeneous clusters. (b) Melting point of homogeneous clusters versus their radii.

observed for the binary systems. However, in the range 50500 the variation of the melting point seems less drastic than in the smaller size range, even though numerous sizes are obviously missing from the reported data. The bulk limit for the melting point is seen to be 0.039 .

This observation should not obscure the possibility that even large sizes can exhibit a multiple-stage melting. For example, significant premelting peaks have been reported in large atomic clusters [55]. The results obtained for $n=500$ show a similar feature. Even though some premelting peaks are present in the caloric curves of large clusters, a major peak seems to dominate above about 100 ions. This result may suggest that the transition between the finite size regime and bulk [36] takes place near this size.

\section{DISCUSSION AND CONCLUSION}

In this work, we have investigated some dynamic and thermodynamic properties of finite 2D charged particles confined by a parabolic trap. Our extensive data on homogeneous and heterogeneous clusters were obtained from MD and $\mathrm{MC}$ simulations. These data were interpreted by visualizing the energy landscape through disconnectivity graphs. Previous experimental and numerical findings suggest that there are three types of motion for a particle: it can oscillate, rotate in a relatively regular orbit, or make jumps between shells, possibly causing isomerizations. These various phenomena were also observed in the present work, but we found evidence that additional "premelting" events due to structural transitions may also occur. The various stages of melting create ambiguities in defining the melting point, especially in small clusters.

Firstly, rotational motion is facile when very small energy barriers separate the global energy minimum from itself, as is apparent from the disconnectivity graphs for the 17- and 21particle homogeneous clusters, or the $\{4,5\}$ binary cluster. Internal rotation may give rise to significant jumps in the RMS bond-length fluctuation, sometimes above 0.1. Radial melting is then seen as yet another increase in $\delta$ or other dynamical indicators at higher energy. Therefore, the Lindemann criterion $(\delta \geq 0.1)$ is not fully reliable for detecting melting.

Secondly, rotational melting has no thermodynamic consequence since only a single isomer is involved. The thermodynamics is sensitive to the presence of different isomers, and also to the intrinsic shape of the potential felt by the particles. Irrespective of the isomers, the heat capacity has a natural drop over a rather broad temperature range, which marks the continuous transition toward the gas phase. Hence, even a single-isomer cluster can have heat capacities with complex temperature dependence, as exemplified by the small $\{4,5\}$ binary clusters.

Thirdly, several isomers can give rise to preliminary peaks in the heat capacity, which may sometimes obscure the features associated with full radial melting. We found such a case for the 31-particle cluster interacting through dipolar forces, but very large clusters can also exhibit particularly strong premelting peaks. The structural transition occurring in the 31-particle cluster has the same origins as the fccicosahedral transition in the 3D 38-atom Lennard-Jones cluster [51,52]: the second morphology has a much larger entropy than the global minimum. This phenomenon has important consequences for the constant energy dynamical behavior, as this transition is not seen until sufficient energy to surmount the isomerization barrier is present. Thus there is a finite energy range where MD simulations starting from the ground state structure are nonergodic, which is reflected by a strong shift in the estimated melting temperature.

Most of our results illustrate how a simple and unique picture of melting is hampered by finite size effects. Both the dynamical parameters and the thermodynamical curves contribute to explaining related but different aspects of melting in these 2D clusters. In particular, our work does not support a single parameter as a universal measure for the melting point that could be used unambiguously for all clusters. Nevertheless, the landscape approach [27] that has been followed 
here was found to provide useful insight for interpreting and understanding the various numerical data.

The data gathered here for a broad range of sizes would seem to prevent a general classification on the phenomenology of melting to be made. For example, the choice of molecular dynamics or Monte Carlo method, and the microcanonical or canonical ensemble, can change the observed behavior significantly, particularly at small sizes where the differences between the two statistical ensembles are the largest. For instance, some structural transitions might not appear with conventional MD methods, due to the disconnected character of phase space at low total energies.

However, three main categories of behavior can be inferred from our results. Especially in small clusters, a twostep orientational-then-radial melting has been observed, with clear dynamical signatures, but only a weak thermodynamical signature. Clusters exhibiting structural transitions, on the other hand, may not involve any rotational melting but display a strong change in their caloric curve. Finally, a more conventional single-step melting is sometimes seen, as expected in larger clusters, and also for smaller "magic" sizes.
Our calculations indicate that significant finite size effects remain in the thermodynamics of the largest clusters considered. However, above a few hundred ions, melting seems to be essentially a one-step process. This result is consistent with the expectation that the dynamics become more and more dominated by localized processes, due to the less favorable rotational motion. This observation seems to justify the use of the Lindemann index or other dynamical parameters for larger systems. Additionally, the main heat capacity peak becomes larger, and its maximum value coincides with the onset of disorder in the dynamical indicators. Even though premelting features may remain noticeable, we expect the onset of radial melting to become closer and closer to orientational melting, and eventually similar to a bulk first-order melting process.

\section{ACKNOWLEDGMENT}

We are grateful for a collaborative research grant given by CNRS and TUBITAK.
[1] Yu. E. Lozovik and V. A. Mandelshtam, Phys. Lett. A 145, 269 (1990).

[2] Yu. E. Lozovik and V. A. Mandelshtam, Phys. Lett. A 165, 469 (1992).

[3] V. M. Bedanov and F. M. Peeters, Phys. Rev. B 49, 2667 (1994).

[4] V. A. Schweigert and F. M. Peeters, Phys. Rev. B 51, 7700 (1995).

[5] F. M. Peeters, V. A. Schweigert, and V. M. Bedanov, Physica B 212, 237 (1995).

[6] B. Partoens and F. M. Peeters, J. Phys.: Condens. Matter 9, 5383 (1997).

[7] V. A. Schweigert and F. M. Peeters, J. Phys.: Condens. Matter 10, 2417 (1998).

[8] L. Cândido, J.-P. Rino, N. Studart, and F. M. Peeters, J. Phys.: Condens. Matter 10, 11627 (1998).

[9] V. A. Schweigert, I. V. Schweigert, A. Melzer, A. Homann, and A. Piel, Phys. Rev. Lett. 80, 5345 (1998).

[10] W.-T. Juan, Z.-H. Huang, J.-W. Hsu, Y.-J. Lai, and L. I, Phys. Rev. E 58, R6947 (1998).

[11] A. A. Koulakov and B. I. Shklovskii, Phys. Rev. B 57, 2352 (1998).

[12] Yu. E. Lozovik and E. A. Rakoch, Phys. Lett. A 240, 311 (1998).

[13] Y.-J. Lai and L. I, Phys. Rev. E 60, 4743 (1999).

[14] I. V. Schweigert, V. A. Schweigert, and F. M. Peeters, Phys. Rev. Lett. 84, 4381 (2000).

[15] M. Kong, B. Partoens, and F. M. Peeters, Phys. Rev. E 65, 046602 (2002).

[16] J. A. Drocco, C. J. Olson Reichhardt, C. Reichhardt, and B. Jankó, Phys. Rev. E 68, 060401(R) (2003).

[17] A. Melzer, A. Homann, and A. Piel, Phys. Rev. E 53, 2757 (1996)

[18] R. Bubeck, C. Bechinger, S. Neser, and P. Leiderer, Phys. Rev.
Lett. 82, 3364 (1999).

[19] M. Saint Jean, C. Even, and C. Guthmann, Europhys. Lett. 55, 45 (2001).

[20] A. Melzer, Phys. Rev. E 67, 016411 (2003).

[21] R. Ichiki, Y. Ivanov, M. Wolter, Y. Kawai, and A. Melzer, Phys. Rev. E 70, 066404 (2004).

[22] P. Leiderer, J. Low Temp. Phys. 87, 247 (1992).

[23] C. Sikorski and U. Merkt, Phys. Rev. Lett. 62, 2164 (1989); R. C. Ashoori, H. L. Stormer, J. S. Weiner, L. N. Pfeiffer, S. J. Pearton, K. W. Baldwin, and K. W. West, ibid. 68, 3088 (1992).

[24] E. J. Yarmachuk and R. E. Packard, J. Low Temp. Phys. 46, 479 (1982).

[25] F. Chevy, K. W. Madison, and J. Dalibard, Phys. Rev. Lett. 85, 2223 (2000).

[26] K. Zahn, J. M. Mendez, and G. Maret, Phys. Rev. Lett. 79, 175 (1997).

[27] D. J. Wales, Energy Landscapes (Cambridge University, Cambridge, 2003).

[28] J. J. Thomson, Philos. Mag. 7, 237 (1904).

[29] D. J. Wales and A. M. Lee, Phys. Rev. A 47, 380 (1993).

[30] B. Rinn and P. Maass, Phys. Rev. Lett. 86, 4711 (2001).

[31] I. V. Schweigert, V. A. Schweigert, and F. M. Peeters, Phys. Rev. Lett. 86, 4712 (2001).

[32] F. Calvo, in Progress in Chemical Physics Research, edited by A. N. Linke (Nova Science Publishers, Happauge, NY, in press).

[33] H.-P. Cheng and R. S. Berry, Phys. Rev. A 45, 7969 (1992).

[34] J.-B. Maillet, A. Boutin, S. Buttefey, F. Calvo, and A. H. Fuchs, J. Chem. Phys. 109, 329 (1998).

[35] G. A. Breaux, R. C. Benirschke, T. Sugai, B. S. Kinnear, and M. F. Jarrold, Phys. Rev. Lett. 91, 215508 (2003).

[36] F. Calvo and F. Spiegelman, J. Chem. Phys. 112, 2088 (2000).

[37] R. J. Hinde, R. S. Berry, and D. J. Wales, J. Chem. Phys. 96, 
1376 (1992).

[38] C. Amitrano and R. S. Berry, Phys. Rev. Lett. 68, 729 (1992).

[39] E. Yurtsever, Europhys. Lett. 37, 91 (1997).

[40] F. Calvo, J. Chem. Phys. 108, 6861 (1998).

[41] F. Calvo, Phys. Rev. E 58, 5643 (1999); 60, 2771 (2001).

[42] D. J. Wales and R. S. Berry, J. Phys. B 24, L351 (1991).

[43] G. Geyer, in Computing Science and Statistics: Proceedings of the 23rd Symposium on the Interface, edited by E. K. Keramidas (Interface Foundation, Fairfax Station, VA, 1991), p. 156.

[44] O. M. Becker and M. Karplus, J. Chem. Phys. 106, 1495 (1997).

[45] D. J. Wales, M. A. Miller, and T. R. Walsh, Nature, 394, 394 (1998).

[46] Z. Li and H. A. Scheraga, Proc. Natl. Acad. Sci. U.S.A. 84, 6611 (1987).

[47] D. J. Wales and J. P. K. Doye, J. Phys. Chem. A 101, 5111
(1997).

[48] G. Benettin, L. Galgani, and J.-M. Strelcyn, Phys. Rev. A 14, 2338 (1974).

[49] L. Casetti, R. Livi, and M. Pettini, Phys. Rev. Lett. 74, 375 (1995).

[50] F. Calvo, J. P. K. Doye, and D. J. Wales, J. Chem. Phys. 116, $2642(2002)$.

[51] J. P. K. Doye, M. A. Miller, and D. J. Wales, J. Chem. Phys. 110, 6896 (1999).

[52] J. P. Neirotti, F. Calvo, D. L. Freeman, and J. D. Doll, J. Chem. Phys. 112, 10340 (2000).

[53] P. Labastie and R. L. Whetten, Phys. Rev. Lett. 65, 1567 (1990).

[54] V. N. Ryzhov and E. E. Tareyeva, Phys. Rev. B 51, 8789 (1995).

[55] F. Calvo and F. Spiegelman, J. Chem. Phys. 120, 9684 (2004). 